\title{
Métodos de escarificación en semillas de Guaiacum coulteri, especie amenazada del bosque tropical caducifolio del norte de Sinaloa, México
}

\section{Scarification methods in seeds of Guaiacum coulteri, an endangered species that inhabits the tropical deciduous forest of northern Sinaloa, Mexico}

\author{
Bardo Heleodoro Sánchez-Soto ${ }^{1 *}$, Elizabeth Pacheco-Aispuro², Gabriel Antonio Lugo-García ${ }^{1}$, \\ Álvaro Reyes-Olivas ${ }^{1}$ \& Edmundo García-Moya ${ }^{3}$
}

${ }^{1}$ Universidad Autónoma de Sinaloa, Facultad de Agricultura del Valle del Fuerte. Calle 16 y Avenida Japariqui. Juan José Ríos, Ahome, Sinaloa, México. C. P. 81110.

2Departamento Académico de Ciencias Biológicas, Universidad de Occidente, Unidad Los Mochis. Blvd. Macario Gaxiola y Carretera internacional México 15. Los Mochis, Sinaloa, México. C. P. 81223.

${ }^{3}$ Colegio de Postgraduados, Posgrado en Botánica. Km 36.5 Carretera México-Texcoco, Montecillo, Texcoco, Estado de México. México. C.P. 56230.

*bardosanchez@hotmail.com

\begin{abstract}
RESUMEN
Esta investigación documentó el efecto de métodos de escarificación física y química en semillas de Guaiacum coulteri, especie del bosque tropical caducifolio sinaloense protegida por leyes mexicanas e internacionales, con la finalidad de contribuir a su conocimiento germinativo. Para ello se evaluaron seis tratamientos pregerminativos sobre el tiempo de inicio de germinación (TIG), porcentaje inicial de germinación (PIG), tiempo medio de germinación $\left(t_{50}\right)$ y porcentaje final de germinación (PFG) con un diseño completamente al azar bajo condiciones de laboratorio. Los métodos de escarificación influyeron de manera significativa $(p<0,05)$ en las cuatro variables estudiadas. Las semillas sometidas a los tratamientos $\mathrm{H}_{2} \mathrm{SO}_{4}$ por 5 y 10 min iniciaron su germinación un día después de la siembra, mientras que en el testigo ocurrió hasta el tercer día. El porcentaje inicial de germinación se incrementó al 73,3\% con el tratamiento de lija versus el testigo que fue de $10 \%$. La escarificación con $\mathrm{H}_{2} \mathrm{SO}_{4}$ por 5 y 10 min adelantaron el $t_{50}$ a los $1,8 \mathrm{~d}$, en contraste con el testigo que fue a los 5,2 d. El mayor porcentaje final de germinación se obtuvo con el lijado de semillas (86,7\%), a diferencia del 51,1\% alcanzado en agua caliente $\left(60^{\circ} \mathrm{C}\right)$ durante $10 \mathrm{~min}$. Los conocimientos derivados de este ensayo demostraron que los métodos de escarificación con lija y ácido sulfúrico fueron los mejores tratamientos pregerminativos, debido a que fragmentaron o debilitaron la testa de las semillas y permitieron obtener altas tasas de germinación, lo que tendrá relevancia para la producción de plántulas.
\end{abstract}

Palabras clave: Conservación, guayacán, latencia física, semilla, tratamientos pregerminativos.

\begin{abstract}
This investigation documented the effect of methods of physical and chemical scarification in seeds of Guaiacum coulteri, a species of tropical deciduous forest from Sinaloa protected by national and international laws, in order to contribute to the germinative knowledge of the species. For this, six pregerminative treatments were evaluated on the initial germination time (IGT), initial germination percentage (IGP), mean germination time $\left(t_{50}\right)$ and final germination percentage (FGP) with a randomized completely design under laboratory conditions. Scarification methods influenced significantly $(p<0.05)$ in the four variables. The seeds subjected to the treatments $\mathrm{H}_{2} \mathrm{SO}_{4}$ for 5 and $10 \mathrm{~min}$ started to germinate one day after sowing, whereas in the control occurred until the third day. The initial germination percentage increased to $73.3 \%$ with the treatment of sandpaper against the control that was just $10 \%$. The scarification with $\mathrm{H}_{2} \mathrm{SO}_{4}$ for 5 and 10 min advanced the mean germination time to $1.8 \mathrm{~d}$, in relation with the control that was $5.2 \mathrm{~d}$. The higher final germination percentage was obtained with the sanding of the seeds $(86.7 \%)$, unlike $51.1 \%$ achieved in hot water $\left(60{ }^{\circ} \mathrm{C}\right)$ for $10 \mathrm{~min}$. The knowledge derived from this essay demonstrated that the methods of scarification with sandpaper and sulfuric acid were the best pregerminative treatments, due to they fragmented or weakened the seed coat and allowed obtaining high germination rates, what will have relevance for the production of seedlings.
\end{abstract}

KEYWORDS: Conservation, guayacán, physical dormancy, pregerminative treatments, seed. 


\section{INTRODUCCIÓN}

La mayoría de las especies que habitan en los bosques tropicales caducifolios tienen semillas con latencia física (Baskin \& Baskin 1998, Khurana \& Singh 2001), lo que les permite formar reservorios de semillas (Álvarez-Aquino et al. 2014, Soriano et al. 2014). Una alternativa para eliminar esta condición en semillas de algunas especies es la escarificación física, química o la aplicación de agua caliente al promover que la testa u otras estructuras sean permeables al agua y aire, de tal modo que estimulen la germinación (Baskin \& Baskin 1998, Khurana \& Singh 2001). La escarificación mecánica se ha utilizado mediante la fricción de la semilla con papel lija o corte de la testa con ayuda de navaja en distintas especies de leguminosas (Tigabu \& Odén 2001, Álvarez-Aquino et al. 2014, Bushman et al. 2015). Los tratamientos pregerminativos químicos consisten en la inmersión de las semillas, por un periodo de tiempo, a sustancias corrosivas, entre ellas el ácido sulfúrico o ácido clorhídrico concentrado como se ha informado por distintos investigadores (GodínezÁlvarez \& Flores-Martínez 1999, Baskin \& Baskin 2004, Soriano et al. 2014). El ablandamiento de la testa de la semilla con ayuda de agua caliente se ha demostrado que también influye en la ruptura de latencia física de las semillas (Godínez-Álvarez \& Flores-Martínez 1999, Sánchez-Paz \& Ramírez-Villalobos 2006). Los estudios de germinación en especies de Guaiacum son limitados (Taylor 1966, Everitt 1983, Pérez 1996, González-Rivas et al. 2009). Taylor (1966) demuestra que las semillas de Guaiacum sanctum L. tienen latencia física provocada por el arilo y la testa de la semilla, debido a que contienen compuestos que inhiben la germinación; una vez que se remueve el arilo y la testa, alrededor del $100 \%$ de las semillas logran germinar en un periodo de 2 a 5 días, mientras que en el testigo no germinan. Everitt (1983) evalúa el efecto de la temperatura en las semillas de Guaiacum angustifolium Engelm., alcanzando entre el 87 y $92 \%$ de germinación a temperaturas de 25 y $30{ }^{\circ} \mathrm{C}$; situación semejante en semillas de G. sanctum, que logran germinar cerca del $80 \%$ al ser incubadas a temperaturas de 20 o $25{ }^{\circ} \mathrm{C}$ (González-Rivas et al. 2009). Pérez (1996) aplica tratamientos pregerminativos de inmersión en ácido sulfúrico y la imbibición en agua a semillas de Guaiacum officinale L., con eficiencias de aproximadamente $90 \%$ de germinación promedio, mayor velocidad promedio de germinación y menor periodo de germinación.

Guaiacum coulteri A. Gray, conocido como guayacán, es un arbusto o árbol que mide hasta $12 \mathrm{~m}$ de altura (Fig. 1A, 1B y 1C). Se distribuye en bosques tropicales caducifolios de la costa del Pacífico Mexicano. Los estados de Oaxaca, Jalisco, Sinaloa y Sonora ocupan el 66\% de la superficie donde se le encuentra (López-Toledo et al. 2011). La especie esta amenazada de acuerdo con la NOM-059-
SEMARNAT-2010, se cita en la Lista Roja de Especies Amenazadas de la Unión Internacional para la Conservación de la Naturaleza (IUCN, por sus siglas en inglés) (GonzálezEspinosa 1998), mientras que en 2016 se incluyó en el apéndice II de la Lista de Especies de la Convención sobre el Comercio Internacional de Especies Amenazadas de Fauna y Flora Silvestres (CITES, por sus siglas en inglés). El principal aprovechamiento de Guaiacum L. es maderable, por lo que las especies del género han sido sujetas a derriba intensa y constante durante periodos prolongados, lo que ha generado un repoblamiento natural insuficiente. En particular, G. coulteri presenta una baja densidad de árboles de talla comercial que limita su aprovechamiento (Gordon et al. 2005).

La información sobre el comportamiento germinativo de Guaiacum coulteri, es escasa o nula. Sólo se tiene el informe de Coatzín-Ramírez (2004), quién indica que las semillas cosechadas en los municipios de Navolato, Culiacán y El Fuerte en Sinaloa, México, al ser tratadas con ácido sulfúrico al $96 \%$ por ocho minutos de remojo, alcanzan el $70 \%$ de germinación a los siete días de la siembra. Con base en lo precedente, esta investigación evalúa el efecto de métodos de escarificación física y química sobre el proceso de germinación de semillas de G. coulteri, especie amenazada del bosque tropical caducifolio sinaloense de México, bajo el supuesto de que al menos uno de los tratamientos pregerminativos será apropiado para asegurar una alta tasa de germinación y, por ende, la producción de plántulas que puedan ser utilizadas en programas de repoblamiento de la especie.

\section{MATERIALES Y MÉTODOS}

Material biolóGico

Se utilizó semillas de frutos maduros cosechados en poblaciones vegetales de la localidad El Realito, Municipio El Fuerte, Sinaloa, México, en el mes de septiembre del 2011. Las semillas se extrajeron de los frutos y se almacenaron en bolsas de papel estraza en el herbario de la Facultad de Agricultura del Valle del Fuerte, Universidad Autónoma de Sinaloa, a temperatura ambiente $\left(\approx 25^{\circ} \mathrm{C}\right) \mathrm{y}$ humedad relativa menor a $50 \%$, hasta dar inicio el ensayo.

ENSAYOS DE GERMINACIÓN

Se evaluó el efecto de la escarificación en la ruptura de latencia física de las semillas mediante un diseño completamente aleatorio con tres repeticiones. La unidad experimental o repetición de cada tratamiento estuvo representada por 30 semillas que se sembraron sobre toallas absorbentes humedecidas con $5 \mathrm{ml}$ de agua destilada en cajas Petri de $8 \mathrm{~cm}$ de diámetro. Antes de efectuar los ensayos, las semillas se desinfectaron durante 1 min en una solución de fungicida Captan 50, marca BRAVOAG ${ }^{\circledR}$. 


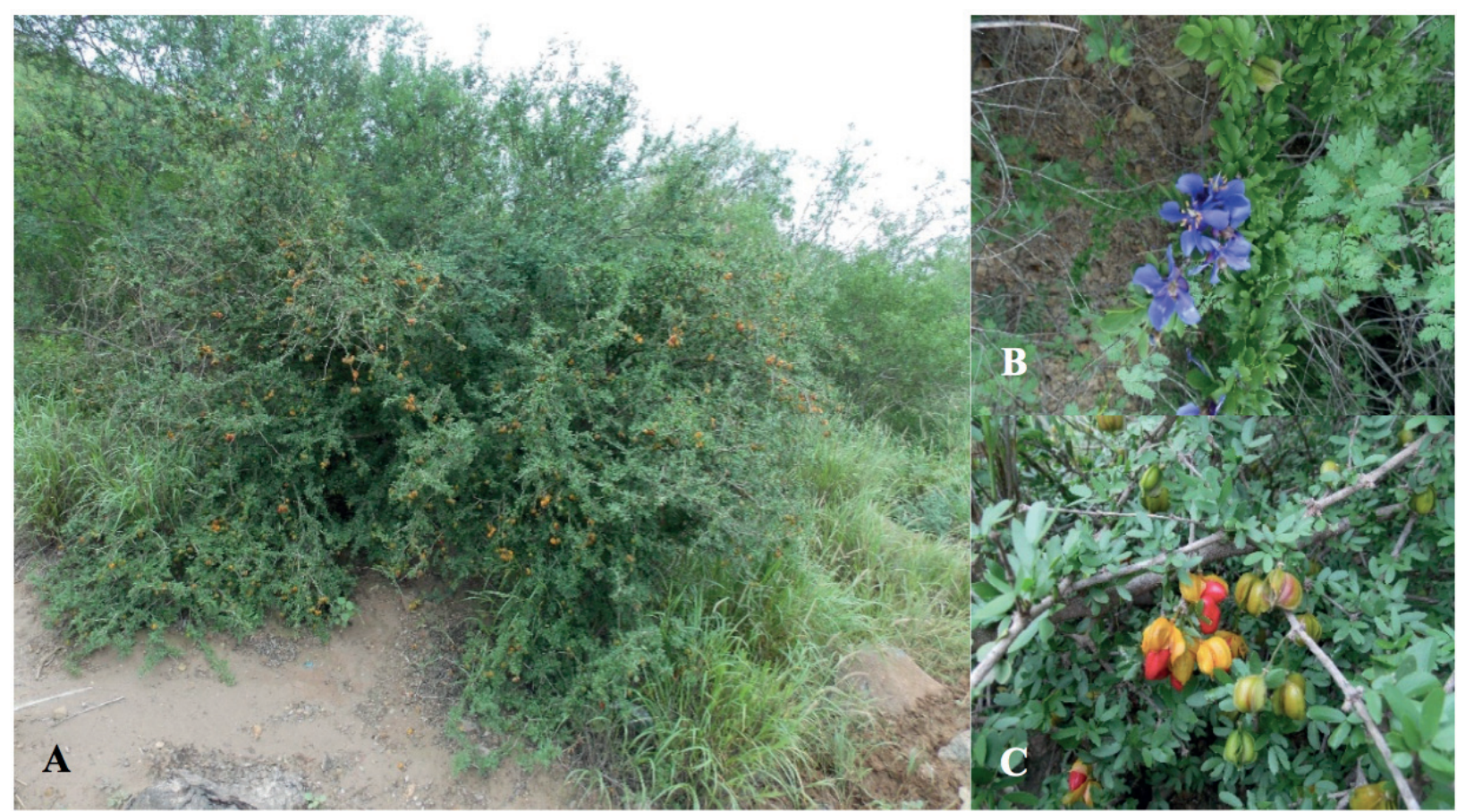

Figura 1. Ejemplar de Guaiacum coulteri A. Gray en un bosque tropical seco del norte de Sinaloa (A), flores (B), frutos y semillas (C). / Specimen of Guaiacum coulteri A. Gray in a tropical dry forest of northern Sinaloa (A), flowers (B), fruits and seeds (C).

Los métodos de escarificación para fragmentar o debilitar la testa consistieron en: semillas sin escarificar o testigo (T1), fricción con lija (T2) para madera K-61 (Marca Fandeli), inmersión en $\mathrm{H}_{2} \mathrm{SO}_{4}$ por periodos de 1 (T3), 5 (T4) y $10 \mathrm{~min}$ (T5), e imbibición en agua caliente a temperatura de $60{ }^{\circ} \mathrm{C}$ por $10 \mathrm{~min}$ (T6). Los ensayos de germinación se realizaron en el Laboratorio de Química de la Universidad de Occidente a temperaturas constantes día/noche de 27 $\pm 1{ }^{\circ} \mathrm{C}$ y fotoperiodo de $12 \mathrm{~h}$ provisto por una lámpara de luz fluorescente de $22 \mathrm{~W}$. El recuento diario de semillas germinadas se llevó a cabo durante ocho días después de la siembra, periodo en el cual se logró la máxima germinación en todos los tratamientos. El criterio para considerar una semilla germinada fue la emergencia y longitud de $2 \mathrm{~mm}$ de la radícula.

\section{ANÁLISIS DE LOS DATOS}

El porcentaje final de germinación ( $\mathrm{PFG})$, en relación al número de semillas colocadas en cada repetición (30 semillas/ repetición), se estimó por unidad experimental en cada tratamiento. Además, se calculó el tiempo de inicio de germinación (TIG), día en que se registró la primera o primeras semillas germinadas, porcentaje inicial de germinación (PIG) y tiempo medio de germinación $\left(t_{50}\right)$ de acuerdo con Ellis \& Roberts (1978), tiempo en que se alcanza el $50 \%$ de las semillas germinadas.

Las variables de respuesta se sometieron a pruebas de normalidad con el estadístico de Shapiro \& Wilk (1965); el porcentaje final de germinación (PFG) y el tiempo medio de germinación $\left(t_{50}\right)$ mostraron normalidad en sus datos, por lo que se sometieron al análisis de varianza de una vía, mientras que la separación de medias se realizó con la prueba de rangos estudentizados de Tukey al 5\%. Las variables tiempo de inicio de germinación (TIG) y porcentaje inicial de germinación (PIG) no se ajustaron a la distribución normal, aun con alguna transformación, así que se analizaron con el ANOVA no paramétrico de KruskalWallis y como estadístico de prueba fue la aproximación a la $\chi^{2}$ de Pearson, con un nivel de significancia de 5\%, mientras que para demostrar diferencias significativas entre métodos de escarificación se utilizó la prueba de la Suma de Rangos de Wilcoxon para todos los pares posibles de tratamientos. El análisis estadístico se realizó con los procedimientos UNIVARIATE, GLM y NPAR1WAY que están disponibles en el programa SAS v. 9.2 (SAS Institute 2009).

\section{RESULTADOS}

Los porcentajes de inicio de germinación de las semillas de Guaiacum coulteri mostraron diferencias estadísticas $\left(\chi^{2}=13,735 ; p=0,0174\right.$; Kruskal-Wallis) entre los métodos de escarificación (Fig. 2A); el porcentaje inicial de germinación mayor ocurrió en semillas lijadas con $73,3 \%$, mientras que el testigo tuvo el menor porcentaje de inicio de germinación con $10,0 \%$. En los porcentajes finales de germinación 

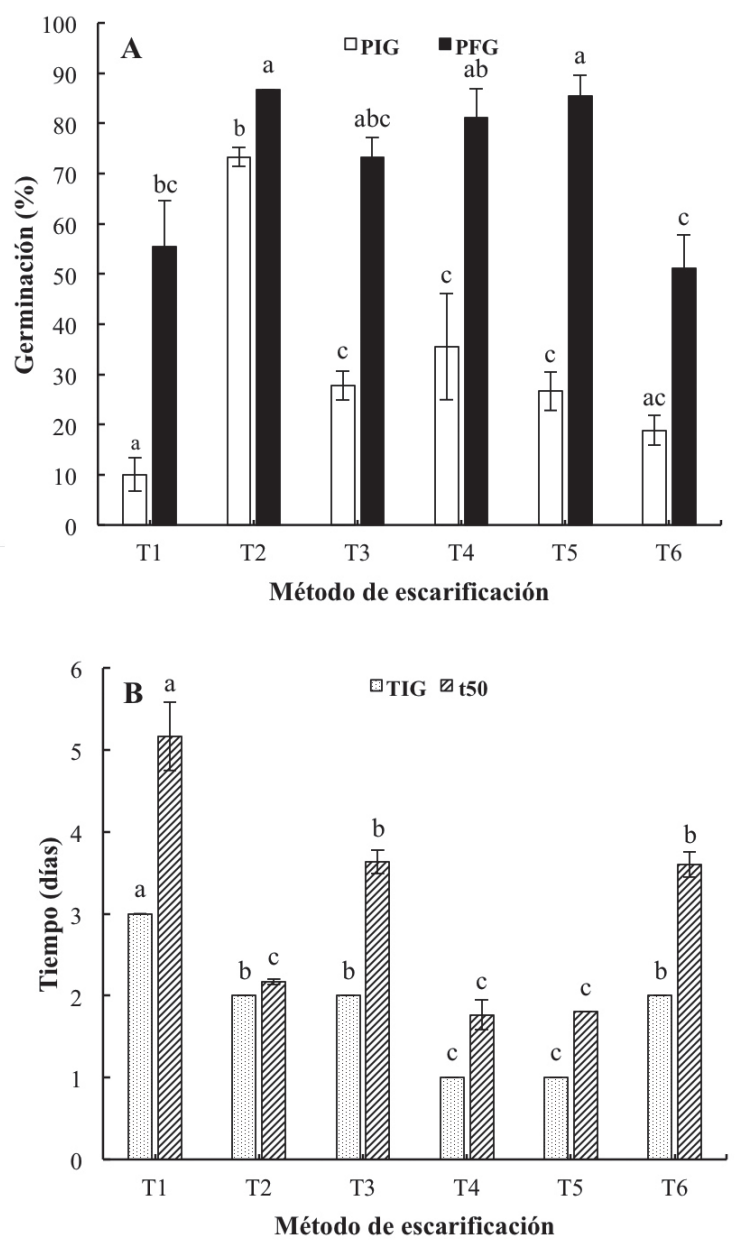

Figura 2. A. Porcentaje inicial de germinación (PIG; barras blancas), porcentaje final de germinación (PFG; barras negras). B. Tiempo de inicio de germinación (TIG; barras con puntos) y tiempo medio de germinación $\left(t_{50}\right.$; barras con rayas) de semillas de Guaiacum coulteri A. Gray obtenidos después de aplicar un método de escarificación. Testigo (T1), lija (T2), $\mathrm{H}_{2} \mathrm{SO}_{4}$ por $1 \mathrm{~min}$ (T3), $\mathrm{H}_{2} \mathrm{SO}_{4} 5 \mathrm{~min}$ (T4), $\mathrm{H}_{2} \mathrm{SO}_{4} 10 \mathrm{~min}$ (T5) y agua caliente $\left(60^{\circ} \mathrm{C}\right)$ 10 min (T6). Se indican las medias \pm E.E. ( $n=3$ cajas Petri con 30 semillas cada una). Para cada panel, letras distintas indican diferencias estadísticas $(p<0,05)$ del análisis de varianza de una vía para el porcentaje final de germinación y tiempo medio de germinación y del análisis Kruskal-Wallis para el tiempo de inicio de germinación y porcentaje de inicio de germinación seguido de los métodos de Tukey o Wilcoxon, respectivamente. / Initial germination percentage (IGP; white bars), final germination percentage (FGP; black bars) (A), initial germination time (IGT; dotted bars) and mean germination time $\left(t_{50}\right.$; lined bars) (B) of the germination the seeds of Guaiacum coulteri A. Gray obtained after applying a scarification method: control (T1), sandpaper (T2), $\mathrm{H}_{2} \mathrm{SO}_{4}$ for 1 min (T3), $\mathrm{H}_{2} \mathrm{SO}_{4} 5$ min (T4), $\mathrm{H}_{2} \mathrm{SO}_{4} 10 \mathrm{~min}$ (T5) and hot water $\left(60^{\circ} \mathrm{C}\right) 10 \mathrm{~min}(\mathrm{~T} 6)$. The means are shown \pm E.E. ( $n=3$ Petri dish with 30 seeds for each one). Per each panel, different letters indicate statistical differences $(p<0.05)$ of the oneway classification for the final germination percentage and mean germination time and the Kruskal-Wallis analysis for the initial germination time and initial germination percentage followed by the methods of Tukey or Wilcoxon, respectively. también existen diferencias significativas $(\mathrm{F}=7,39$; $p=0,0022$; ANOVA de una vía) entre los tratamientos (Fig. 2A); el mayor porcentaje final de germinación lo estimuló la frotación de las semillas con lija $(86,7 \%)$, en comparación con el menor porcentaje final de germinación de 51,1\% que ocurrió con agua caliente $\left(60^{\circ} \mathrm{C}\right)$ durante $10 \mathrm{~min}$. El comportamiento del porcentaje de germinación acumulado que se obtuvo por día después de aplicar un método de escarificación se describe en la Figura 3.

Los tiempos de inicio de germinación de las semillas de $G$. coulteri difirieron de manera significativa $\left(\chi^{2}=17,00\right.$; $p=0,0045$; Kruskal-Wallis) entre tratamientos (Fig. 2B). Esto es, las semillas inmersas durante 5 y 10 min en $\mathrm{H}_{2} \mathrm{SO}_{4}$ fueron las primeras en germinar (un día después de haberlas sembrado). Las semillas sujetas a escarificación con lija, las inmersas en $\mathrm{H}_{2} \mathrm{SO}_{4}$ por 1 min y las embebidas en agua caliente $\left(60{ }^{\circ} \mathrm{C}\right)$ durante $10 \mathrm{~min}$ mostraron la radícula al segundo día. En contraste, las semillas del testigo demoraron hasta el tercer día para manifestar su germinación. El tiempo medio de germinación registró diferencias estadísticas ( $\mathrm{F}=42,96 ; p<0,0001$; ANOVA de una vía) entre los distintos métodos de escarificación (Fig. 2B). Las diferencias se manifestaron entre el testigo y el resto de los tratamientos. La inmersión en $\mathrm{H}_{2} \mathrm{SO}_{4}$ por 5 y 10 min presentó un tiempo medio de germinación de 1,8 días, en cambio el tiempo medio de germinación del testigo se demoró hasta los 5,2 días. También existen diferencias significativas entre los tratamientos de fricción con lija, $\mathrm{H}_{2} \mathrm{SO}_{4}$ por 5 y $10 \mathrm{~min}(2,2$ d, 1,8 d y 1,8 d, respectivamente) versus las aplicaciones de $\mathrm{H}_{2} \mathrm{SO}_{4}$ por $1 \mathrm{~min}(3,7 \mathrm{~d})$ o agua caliente $\left(60^{\circ} \mathrm{C}\right)$ durante 10 $\min (3,6 d)$.

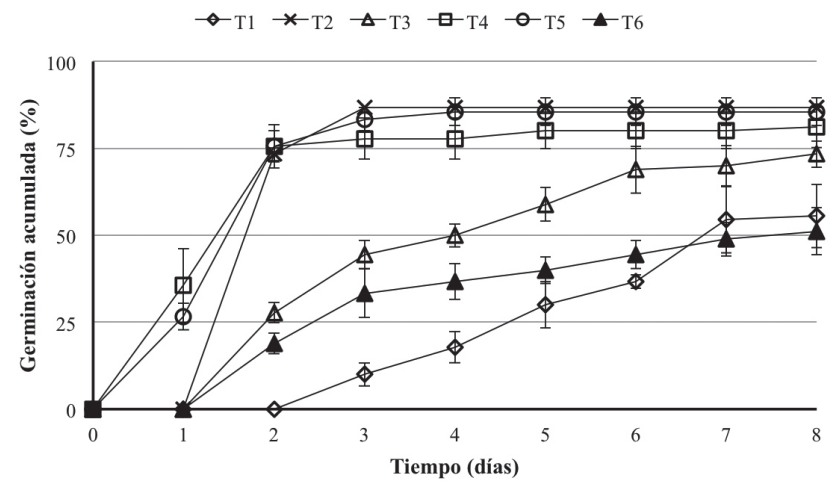

FIgURA 3. Porcentaje de germinación acumulada de Guaiacum coulteri A. Gray bajo diferentes métodos de escarificación: testigo (T1), lija (T2), $\mathrm{H}_{2} \mathrm{SO}_{4}$ por $1 \mathrm{~min}$ (T3), $\mathrm{H}_{2} \mathrm{SO}_{4} 5 \mathrm{~min}$ (T4), $\mathrm{H}_{2} \mathrm{SO}_{4}$ $10 \mathrm{~min}$ (T5) y agua caliente $\left(60^{\circ} \mathrm{C}\right) 10 \mathrm{~min}(\mathrm{~T} 6)$. Barras verticales representan el error estándar de la media. / Cumulative germination percentages of Guaiacum coulteri A. Gray under different method of scarification: control (T1), sandpaper (T2), $\mathrm{H}_{2} \mathrm{SO}_{4}$ for $1 \mathrm{~min}$ (T3), $\mathrm{H}_{2} \mathrm{SO}_{4} 5 \mathrm{~min}$ (T4), $\mathrm{H}_{2} \mathrm{SO}_{4} 10 \mathrm{~min}$ (T5) and hot water $\left(60^{\circ} \mathrm{C}\right)$ $10 \mathrm{~min}$ (T6). Vertical bars represent the standard error of mean. 


\section{DISCUSIÓN}

Los hallazgos de este ensayo demuestran que los métodos de fricción con papel lija e inmersión en ácido sulfúrico eliminan la latencia física de las semillas de Guaiacum coulteri, al influir de manera directa en el tiempo de inicio de germinación, el porcentaje inicial de germinación, el tiempo medio de germinación y el porcentaje final de germinación. Esto es, los tratamientos pregerminativos inducen que la testa de las semillas se adelgace, ablande o agriete, lo que facilita el ingreso de agua e intercambio de gases al embrión, obteniéndose 80,0 y $86,7 \%$ de semillas germinadas al aplicarles ácido sulfúrico o escarificación con lija, respectivamente. Lo anterior se ha demostrado en distintas especies vegetales, principalmente, en leguminosas, entre ellas, las semillas de Mimosa foliolosa Benth. que germinan más del $80 \%$ bajo escarificación mecánica o química (Silveira \& Fernandes 2006), en semillas de dos especies mexicanas del género Mimosa que al ser escarificadas mecánicamente alcanzan más del 70\% de germinación, mientras que las semillas sin tratamiento germinan del $0-12 \%$ (Montaño-Arias et al. 2015) o bien con la aplicación de ácido sulfúrico durante $15 \mathrm{~min}$ en semillas de Caesalpinia spinosa (Feuillée ex Molina) Kuntze y C. barahonensis Urb. que estimula hasta $100 \%$ de germinación en ambas especies (Rossini-Oliva et al. 2006). El comportamiento germinativo de las semillas de $G$. coulteri a la aplicación de agua caliente $\left(60^{\circ} \mathrm{C}\right)$ durante 10 min no muestra efecto significativo $(p<0,05)$ al compararse con el porcentaje final de germinación registrado en el testigo ( $51.1 \%$ versus $55.6 \%$, respectivamente). Esto difiere a lo registrado en Albizia gummifera (J.F. Gmel.) C.A. Sm. (Tigabu \& Odén 2001), Peltophorum pterocarpum (DC.) Backer ex K. Heyne (Atencio et al. 2003) y Leucaena leucocephala (Lam.) de Wit (Sánchez-Paz \& RamírezVillalobos 2006) que alcanzan porcentajes de germinación mayores al $80 \%$ cuando las semillas se tratan con agua caliente de $80{ }^{\circ} \mathrm{C}$.

El efecto benéfico de la escarificación, no solo se manifiesta en el incremento en el porcentaje final de germinación, sino que también se refleja en la reducción en el tiempo de inicio de germinación, en el aumento del porcentaje inicial de germinación y en la aceleración del tiempo medio de germinación, esto es, las semillas inmersas con $\mathrm{H}_{2} \mathrm{SO}_{4}$ al $96 \%$ y lijadas muestran la radícula, al menos de $2 \mathrm{~mm}$ de longitud, uno o dos días después de su siembra, alcanzando un porcentaje inicial de germinación de 30,0 y $73,3 \%$ en un tiempo medio de germinación de 2,4 y 2,2 días, respectivamente. Los resultados concuerdan con los obtenidos por Coatzín-Ramírez (2004), quien al tratar las semillas de $G$. coulteri con $\mathrm{H}_{2} \mathrm{SO}_{4}$ al $96 \%$ por ocho minutos de remojo, observó la germinación al segundo o tercer día después de la siembra logrando el máximo por ciento $(70 \%)$ a los siete días. Otras especies de Guaiacum tienen una respuesta germinativa semejante. Por ejemplo, las semillas de G. sanctum, al removerles el arilo y la testa, estructuras que contienen los compuestos inhibidores de la germinación, germinan alrededor del $100 \%$ en un periodo de 2 a 5 días (Taylor 1966), mientras que G. officinale registra porcentajes de $\approx 90 \%$ cuando las semillas son sumergidas en $\mathrm{H}_{2} \mathrm{SO}_{4}$ por siete minutos o embebidas en agua durante $24 \mathrm{~h}$, dando inicio el proceso de germinación a los 4,3 y 7,3 días con una velocidad promedio de 15,6 semillas germinadas/ día y 10,4 semillas/día en un periodo promedio de 23,3 y 31,7 días, respectivamente, en el que alcanzan el porcentaje final de germinación (Pérez 1996).

Se ha documentado que las temperaturas óptimas para la germinación de semillas de especies del bosque tropical caducifolio son variantes (Khurana \& Singh 2001, González-Rivas et al. 2009, Montaño-Arias et al. 2015) como se ejemplifica en tres especies del bosque tropical seco de Nicaragua donde las temperaturas constantes son mejores que las temperaturas fluctuantes, ya que los mayores porcentajes de germinación en G. sanctum se alcanzan a 20 ó $25^{\circ} \mathrm{C}$, mientras que para Calycophyllum candidissimum (Vahl) DC. se logra a temperaturas constantes de 20 a 35 ${ }^{\circ} \mathrm{C}$ y Cedrela odorata L. a los 20,25 y $35^{\circ} \mathrm{C}$, a diferencia de las temperaturas fluctuantes donde la germinación de las tres especies fue nula (González-Rivas et al. 2009). A pesar de la falta de investigaciones sobre el efecto de las temperaturas óptimas para la germinación en las especies de guayacán (Guaiacum spp.) se sabe que también entre ellas existen distintos requisitos térmicos. Everitt (1983) indica que las temperaturas óptimas para la germinación de $G$. angustifolium son de 25 y $30{ }^{\circ} \mathrm{C}$ con las cuales alcanza del 87 al 92\%, mientras que las semillas de G. sanctum logran germinar cerca del $80 \%$ al ser tratadas con temperaturas de 20 ó $25^{\circ} \mathrm{C}$ y cero por ciento en temperaturas de $30{ }^{\circ} \mathrm{C}$ (González-Rivas et al. 2009). En este ensayo con semillas de G. coulteri se obtienen porcentajes de $55,6 \%$ en el tratamiento testigo a una temperatura constante de $27 \pm$ $1{ }^{\circ} \mathrm{C}$ de incubación. Lo anterior puede explicarse por la amplitud de temperaturas registradas en el bosque tropical caducifolio de México, las cuales varían de los 18 a los 28 ${ }^{\circ} \mathrm{C}$ de temperatura media anual, lo cual significa que estas comunidades pueden existir en un ámbito muy amplio, aunque la mayor parte de este bosque (57\%) se concentra en áreas con temperaturas que van de 22 a $26{ }^{\circ} \mathrm{C}$ (TrejoVázquez 1999), condiciones térmicas que corresponden al bosque tropical caducifolio sinaloense.

Las semillas de G. coulteri en campo se observan sin arilo y debajo de la copa de las plantas madre, mientras que, bajo condiciones de laboratorio, en los ensayos de germinación, algunas fueron infestadas por hongos o se pudrieron. Se ha descrito que en condiciones naturales existen semillas de G. sanctum en las que el arilo, por lo general, se deteriora rápidamente y es fácil de remover, sobre todo en la época de lluvia, cuando se tienen los frutos 
maduros en campo (Taylor 1966, Wendelken \& Martin 1987). En cambio, las semillas que conservaron arilos secos pueden estar sujetas a la infestación y destrucción por larvas de la familia Olethreutidae (Taylor 1966). La importancia de los arilos en las semillas de G. sanctum se debe al color rojo vistoso, que permite atraer a distintos consumidores, en particular, aves como lo informan Wendelken \& Martin (1987), quienes señalan que los frutos y arilos de G. sanctum son aprovechados por 19 especies de aves, agrupadas en 17 géneros y 8 familias que habitan en los matorrales espinosos de las zonas secas del valle del Motagua, Guatemala, en las que al menos 13 especies son dispersores potenciales, por la capacidad de transportar las semillas lejos de la planta madre con la finalidad de alimentar a los polluelos o volantones. En este sentido, se ha documentado que la relevancia ecológica de la dispersión de las semillas por animales, entre ellos mamíferos y aves, se debe al transporte de las mismas a lugares distantes de la planta madre donde pueden existir micrositios favorables para la germinación y establecimiento de plántulas (Howe \& Smallwood 1982, Samuels \& Levey 2005, Jaganathan et al. 2016, Díaz-Vélez et al. 2017).

Baskin \& Baskin (1998) indican que el $76 \%$ de las especies vegetales de los bosques tropicales caducifolios producen semillas con latencia, de las cuales el $67 \%$ tienen latencia física y se sospecha que el $23 \%$ poseen latencia fisiológica (Khurana \& Singh 2001). En la búsqueda de tratamientos pregerminativos que estimulen la ruptura de la latencia, sean aplicado métodos físicos y químicos, los cuales incrementan las tasas de germinación, con el propósito de producir plantas que se han utilizadas en la recuperación de terrenos deteriorados de los bosques tropicales secos de nuestro país (Godínez-Álvarez \& Flores-Martínez 1999). Por lo que la abrasión con papel lija y la aplicación con ácido sulfúrico son ideales para establecer programas de repoblamiento del guayacán, debido a que se asegura un alto número de semillas germinadas, lo que repercute en la producción de plántulas de Guaiacum coulteri.

\section{CONCLUSIONES}

Los métodos de escarificación considerados en esta investigación, en lo particular, la abrasión con papel lija o inmersión en ácido sulfúrico durante 5 ó 10 min influyen de manera positiva en el comportamiento germinativo de las semillas de Guaiacum coulteri, debido a que fragmentan o debilitan la testa, permitiendo el ingreso de oxígeno y agua al embrión necesarios para la germinación. Los tratamientos pregerminativos físicos o químicos adelantan el tiempo de inicio de germinación (TIG), reducen el tiempo medio de germinación $\left(t_{50}\right)$ e incrementan el porcentaje inicial de germinación (PIG) y porcentaje final de germinación (PFG) del guayacán, lo que garantiza la obtención de altas tasas de germinación en un corto plazo con la finalidad de producir plántulas que puedan ser utilizadas en programas de repoblamiento de la especie amenazada del bosque tropical caducifolio sinaloense de México.

\section{LITERATURA CITADA}

Álvarez-Aquino, C., Barradas-Sánchez, L., Ponce-González, O., Williams-LinerA, G. 2014. Soil seed bank, seed removal, and germination in a seasonally dry tropical forest in Veracruz, Mexico. Botanical Sciences 92(1): 111-121.

Atencio, L., Colmenares, R., Villalobos, M.R., Marcano, D. 2003. Tratamientos pregerminativos en acacia San Francisco (Peltophorum pterocarpum) Fabaceae. Revista de la Facultad de Agronomía (LUZ) 20: 63-71.

BASKIn, J.M., BASKIn, C.C. 1998. Seeds, Ecology, Biogeography, and Evolution of Dormancy and Germination. Academic Press. San Diego. 666 pp.

BASkin, J.M., BASkIN, C.C. 2004. A classification system for seed dormancy. Seed Science Research 14: 1-16.

Bushman, B.S., Johnson, D.A., Connors, K.J., Jones, T.A. 2015. Germination and seedling emergence of three semiarid western North American legumes. Rangeland Ecology \& Management 68(6): 501-506.

CoAtzín-Ramírez, R. 2004. Efecto de aislamiento geográfico en germinación, descripción morfológica y tratamientos pregerminativos de Guaiacum coulteri A. Gray. Tesis. Maestría en Ciencias Forestales. División de Ciencias Forestales, Universidad Autónoma Chapingo. Texcoco, Estado de México, México. 53 pp.

Convención sobre el Comercio Internacional de Especies Amenazadas de Fauna y Flora Silvestres (CITES). 2016. Apéndices I, II y III. URL: https://cites.org/esp/app/ appendices.php. Consulta: 13 de septiembre, 2016.

Díaz-Vélez, M.C., Ferreras, A.E., Silva, W.R., Galetto, L. 2017. Does avian gut passage favour seed germination of woody species of the Chaco Serrano Woodland in Argentina? Botany 95(5): 493-501.

Ellis, R.H., RoBerts, E.H. 1978. Towards a rational basis for testing seed quality. In: Hebblethwaite, P.D. (ed.), Seed Production. pp. 605-636. Butterworth, London.

EvERITT, J.H. 1983. Seed germination characteristics of three woody plant species from south Texas. Journal of Range Management 36(2): 246-249.

Godínez-Álvarez, H., Flores-Martínez, A. 1999. Germinación de semillas de 32 especies de plantas de la costa de Guerrero: su utilidad para restauración ecológica. Polibotánica 11: $1-19$.

GonZÁlez-Espinosa, M. 1998. Guaiacum coulteri. The IUCN Red List of Threatened Species 1998: e.T30847A9582622. URL: http://dx.doi.org/10.2305/IUCN.UK.1998.RLTS. T30847A9582622.en. Descargado el 14 de septiembre de 2016.

GonzÁlez-Rivas, B., Tigabu, M., Castro-Marín, G., Odén, P.C. 2009. Seed germination and seedling establishment of Neotropical dry forest species in response to temperature and light conditions. Journal of Forestry Research 20(2): 99-104. 
Gordon, J.E., González, M.A., Hernández, J.V., Lavariega, R.O., ReYes-García, A. 2005. Guaiacum coulteri: an over-logged dry forest tree of Oaxaca, Mexico. Oryx 39(1): 82-85.

Howe, H.F., Smallwood, J. 1982. Ecology of seed dispersal. Annual Review of Ecology and Systematics 13: 201-228.

Jaganathan, G.K., Yule, K., Liu, B. 2016. On the evolutionary and ecological value of breaking physical dormancy by endozoochory. Perspectives in Plant Ecology, Evolution and Systematics 22: 11-22.

Khurana, E., Singh, J.S. 2001. Ecology of seed and seedling growth for conservation and restoration of tropical dry forest: a review. Environmental Conservation 28(1): 39-52.

López-Toledo, L., Gonzalez-Salazar, C., Burslem, D.F.R.P., Martínez-Ramos, M. 2011. Conservation assessment of Guaiacum sanctum and Guaiacum coulteri: historic distribution and future trends in Mexico. Biotropica 43(2): 246-255.

Montaño-Arias, S.A., Camargo-Ricalde, S.L., Grether, R., DíazPontones, D. 2015. Effect of scarification and temperature on seed germination of two Mexican species of Mimosa (Leguminosae-Mimosoideae). Botanical Sciences 93(3): 649-659.

Norma Oficial Mexicana NOM-059-SEMARNAT-2010. 2010. Protección ambiental-Especies nativas de México de flora y fauna silvestres-Categorías de riesgo y especificaciones para su inclusión, exclusión o cambio-Lista de especies en riesgo.

Pérez, A.J. Del P. 1996. Evaluación de diferentes tratamientos pregerminativos aplicados al Guaiacum officinale (guayacán). En: Mesén, F., Rodríguez, Y., Sánchez, A. (eds.), Memorias Primer Seminario Nacional sobre Mejoramiento Genético y Semillas Forestales. pp. 5660. PROSEFOR-CATIE, Santo Domingo, República Dominicana.

Rossini-Oliva, S., Valdés, B., Andrés, M.C., Márquez-Campona,
F., Bueso-López, M. 2006. Germinación de las semillas en algunas especies americanas de Fabaceae y Bignoniaceae cultivadas en Sevilla (SO España). Lagascalia 26: 119-129.

SÁnchez-PaZ, Y., Ramírez-Villalobos, M. 2006. Tratamientos pregerminativos en semillas de Leucaena leucocephala (Lam.) de Wit. y Prosopis juliflora (Sw.) DC. Revista de la Facultad de Agronomía 23(3): 257-272.

SAmuels, I.A., LeVey, D.J. 2005. Effects of gut passage on seed germination: do experiments answer the questions they ask? Functional Ecology 19(2): 365-368.

SAS Institute Inc. 2009. SAS/STAT User's Guide Release 9.2. Cary, SAS Institute North Carolina, EE.UU. 7869 pp.

Shapiro, S.S., Wilk, M.B. 1965. An analysis of variance test for normality (complete samples). Biometrika 52(3/4): 591611.

Silveira, F.A.O., Fernandes, G.W. 2006. Effect of light, temperature and scarification on the germination of Mimosa foliolosa (Leguminosae). Seed Science and Technology 34: 585592.

Soriano, D., Huante, P., Gamboa-deBuen, A., Orozco-Segovia, A. 2014. Effects of burial and storage on germination and seed reserves of 18 tree species in a tropical deciduous forest in Mexico. Oecologia 174(1): 33-44.

TAYLOR, R.A. 1966. Seed germination and seedling growth of Guaiacum sanctum L. Florida State Horticultural Society 79: 468-470

Tigabu, M., OdÉN, P.C. 2001. Effect of scarification, gibberellic acid and temperature on seed germination of two multipurpose Albizia species from Ethiopia. Seed Science and Technology 29: 11-20.

Trejo-VÁzQuez, I. 1999. El clima de la selva baja caducifolia en México. Investigaciones Geográficas 39: 40-52.

Wendelken, P.W., Martin, R.F. 1987. Avian consumption of Guaiacum sanctum fruit in the arid interior of Guatemala. Biotropica 19(2): 116-121.

Recibido: 30.09 .2016

Aceptado: 27.08 .2017 